\title{
Preface: Biomedical Engineering Research at Manipal Academy of Higher Education and Kasturba Medical College, Mangaluru
}

Manipal Academy of Higher Education (MAHE), formerly known as Manipal University, is an umbrella organization that serves as a university-level institution located in Manipal, Karnataka, India. In 1953, Dr. T.M.A. Pai, a physician by profession, founded India's first private medical school, Kasturba Medical College. In 1957, the Manipal Institute of Technology, an engineering school, was established as part of the ongoing expansion of MAHE. Since then, MAHE has expanded significantly in the educational field by founding universities in Sikkim, Jaipur (India), Dubai (UAE), Malaysia, and more recently in Antigua. MAHE has constituent hospitals at Manipal, Mangaluru, and Bangalore attached to their medical and dental schools. MAHE is a member of the Association of Commonwealth Universities and currently has an enrollment of 28,000 students from 57 countries.

The MAHE offers 218 academic programs in 19 disciplines. The educational programs are offered in the areas of medicine, surgery, epidemiology, basic and applied health sciences, architecture, advanced sciences (e.g., nanoscience and technology, laser application in biology medicine), communication (media), dentistry, engineering, European studies and foreign languages, geopolitics and international relations, hospitality including hotel management, humanities, information sciences, life sciences, management (MBA), medicine, natural sciences, nursing, pharmacy, and regenerative medicine. MAHE also offers doctoral programs on a full-time or parttime basis for different disciplines. Many alumni of MAHE have taken up senior leadership positions in academia and corporations all over the world.

The Manipal Institute of Technology (MIT) has 16 academic departments and awards undergraduate, graduate, and doctoral degrees in engineering. Several faculty members at MIT conduct research in biomedical engineering. Kasturba Medical College campus in Mangaluru is the constituent institute of MAHE established in 1953. Significant biomedical engineering research is also ongoing at Kasturba Medical College Mangaluru. In this issue of Critical Reviews in Biomedical Engineering, we are pleased to highlight four specific projects with biomedical engineering applications to patient diagnosis, modelling, and bioinformatics. We present six scholarly research articles describing some of the key areas of healthcare and engineering research under investigation by the faculty and students at both the Manipal and Mangaluru campuses of MAHE.

In the paper, "Estimation of Caffeine Regimens: A Machine Learning Approach for Enhanced Clinical Decision Making in the neonatal intensive care unit (NICU)," Shirwaikar et al. describe the application of machine learning algorithms in estimation of caffeine regimens in preterm neonates with apnea of prematurity in neonatal intensive care units. ${ }^{1}$ Their work is based on the clinical observation that apnea of prematurity is a critical condition observed in most preterm neonates due to inadequate development of the respiratory system. Apart from mechanical ventilation, pharmacotherapy in the form of methylxanthine (caffeine) is used in prevention or reduction of apnea of prematurity in preterm neonates. Adjustment of caffeine dosage is currently performed using pharmacokinetics, a method fraught with difficulties. Shirwaikar et al. have used support vector machine (SVM), decision trees with ensembles created using bagging, boosting, random forest, optimized Multi Layer Perceptron (MLP), and deep learning algorithms to predict the adequacy of caffeine dosage regimens. In a retrospective study of 90 preterm infants, these authors found that an optimized MLP followed by Deep Belief Network (DBN) with Score for Neonatal Acute Physiology (SNAP) is a more accurate model for predicting the therapeutic effectiveness of concentration of caffeine. They also found that the proposed SNAP (illness severity score) acts as a key input variable to enhance the performance of the prediction models. 
Digital images of the cervix can be used for diagnosis of the cancer of the cervix. "Pattern Classification of Images Acquired During Acetic Acid Based Cervical Cancer Screening: A Review," by Vidya Kudva and Keerthana Prasad, describes several image-processing algorithms to analyze cervix images acquired following application of acetic acid in the $\mathrm{OB} / \mathrm{GYN}$ clinic. ${ }^{2}$ During such processing, three attributes (color, vascular patterns, and lesion margins) can help differentiate between normal and abnormal lesions and improve the accuracy of classification algorithms. These authors have reviewed classification algorithms based on the colposcopic and cervicographic images with different features. They follow up their review with a research paper using deep learning algorithms to achieve high accuracy of classification of images acquired in the $\mathrm{OB} / \mathrm{GYN}$ clinic of one of the authors. Their research, reported in the paper "Automation of Detection of Cervical Cancer Using Convolutional Neural Networks," achieves high classification accuracy $(100 \%)$ using the deep learning paradigm. ${ }^{3}$ Automation of image analysis for detection of cervical cancer is very useful, especially if an expert physician is not available on site to interpret such images.

Protein is a macromolecule formed by a chain of amino acids. Protein functions are regulated by its tertiary structure. The prediction of the three-dimensional structure of proteins through experimental methods such as NMR spectroscopy, X-ray crystallography is a time-consuming and tedious process. Hence, it calls for a computational approach to find the structure of a protein. The paper "A Review of Protein Structure Prediction Using Lattice Model" by Sandhya Dubey et al. describes several computational methods used to address the issue. The hydrophobic-polar (HP) model is one of the most widely accepted models to study protein folding and the PSP problem at a coarse level. ${ }^{4}$ Although, the HP model has reduced the complexity of the PSP problem, the possible search space is vast. Dubey et al. provide solutions in the second paper entitled, "A Comparative Study on Single and Multiple Point Crossovers in Genetic Algorithms for Coarse Protein Modeling." A solution to the PSP problem will help in drug design, personalized medication, and other advanced therapeutic strategies.
Body temperature, a complex clinical variable, is one of the oldest vital signs to be measured in human history. A 24-hour record of the body temperature sampled every minute, analyzed using advanced mathematical models, can be used for diagnosis. ${ }^{6}$ In the paper "Classification of Infectious and Noninfectious Diseases Using an Artificial Neural Network from Twenty-Four-Hour Continuous Tympanic Temperature Data of Patients with Undifferentiated Fever," Dakappa et al. classify the undifferentiated fever into two major clinical categories using 24-hour continuous temperature recordings obtained from 103 subjects using an artificial neural network classifier. ${ }^{7}$ They report accuracy of $91.3 \%$ in classifying infectious and noninfectious diseases using ANN. Their study suggests that an analysis of body temperature signal has diverse applications in clinical medicine, circadian physiology, and chronopharmacology and can improve the accuracy of diagnosis in multiple etiologies.

\section{ACKNOWLEDGMENTS}

We thank MAHE for their continuous support in the form of funding, facilities, and making laboratories available for research. We also thank the Government of India support for research in the form of a grant in aid.

\section{REFERENCES}

1. Shirwaikar RD, Acharya DU, Makkithaya K, Surulivelrajan M, Lewis LEU. Estimation of caffeine regimens: a machine learning approach for enhanced clinical decision making at NICU. Crit Rev Biomed Eng. 2018;46(2): 93-115.

2. Kudva V, Prasad K. Pattern classification of images acquired during acetic acid based cervical cancer screening: a review. Crit Rev Biomed Eng. 2018;46(2):117-133.

3. Kudva V, Prasad K, Guruvare S. Automation of detection of cervical cancer using convolutional neural networks. Crit Rev Biomed Eng. 2018;46(2):135-145.

4. Dubey SPN, Kini G, Balaji S, Kumar MS. A review of protein structure prediction using lattice model. Crit Rev Biomed Eng. 2018;46(2):147-162.

5. Dubey SPN, Kini G, Balaji S, Kumar MS. A comparative study on single and multiple point crossovers in genetic algorithms for coarse protein modeling. Crit Rev Biomed Eng. 2018;46(2):163-171. 
6. Dakappa PH, Mahabala C. Analysis of long-term temperature variations in the human body. Crit Rev Biomed Eng. 2015;43(5-6):385-99.

7. Dakappa PH, Prasad K, Mahabala C. Classification of in- fectious and non-infectious diseases using artificial neural network from twenty-four-hour continuous tympanic temperature data of patients with undifferentiated fever. Crit Rev Biomed Eng. 2018;46(2):173-183.

\section{Guest Editors:}

Pradeepa H. Dakappa

Assistant Professor

Department of Pharmacology

World College of Medical Sciences Research and

Hospital, Jhajjar, Haryana, India

Keerthana Prasad

Professor

School of Information Sciences

Manipal University, Manipal, Karnataka, India

Chakrapani Mahabala

Professor

Department of Internal Medicine

Kasturba Medical College, Mangaluru

Manipal Academy of Higher Education, Manipal,

Karnataka, India 
